\title{
HAEMATOMA FOLLOWING EPIDURAL ANAESTHESIA
}

\author{
Albert B. Butler, M.D. and Cloid D. Green, M.D. ${ }^{*}$
}

\begin{abstract}
HAEMoRrhage INTo the spinal epidural space associated with epidural catheterization and anticoagulation represents a rare and serious complication of spinal epidural anaesthesia with three cases ${ }^{1,2,3}$ described in the literature to the present time. Because of the increased use of this form of analgesia, the complication is probably even rarer than the cases reported would indicate. An awareness of this complication is no less compelling because of its potentially serious outcome, and possible remedy by prompt surgery.
\end{abstract}

\section{CASE REPORT}

A 70-year-old man was admitted to the hospital with a thirty-six hour history of numbness of the left foot, which later became cold, cyanotic and mildly painful. There was a history of "cold hands and feet" since 1962. At that time skin and muscle biopsies were performed and a diagnosis of scleroderma was made. Examination revealed thick skin and deformed nails of the fingers and toes. The left foot was cold, cyanotic to the level of the lateral maleolus, and pulses were absent. Pulse was present in the posterior tibial artery on the right. Pulses in the femoral and popliteal arteries were present and normal bilaterally.

Before left anterior and posterior tibial embolectomy, epidural analgesia was induced using an epidural catheter inserted at the $\mathrm{L}-2-3$ space and 2 per cent lidocaine. At the point of inserting the catheter no abnormal bleeding was noted. During the operation 10,000 units of heparin were given intravenously. The interval between catheterization and heparinization was about sixty minutes. Sympathetic blockade was maintained postoperatively by intermittent use of epidural lidocaine 1 per cent. Heparinization was continued as well. Clotting times were 20.5 minutes and 32 minutes at three and seven hours postoperatively. Thirteen hours after operation the epidural catheter was removed, as no further benefit was being obtained from sympathetic blockade. Clotting time was 24.5 minutes at that time. At about 24 hours after operation the patient began to complain of severe back pain. Examination by a house officer revealed no neurological deficit. Locally applied heat produced relief. At 25 hours postoperatively, the patient complained of numbness in both hands and the right leg, unassociated with back or extremity pain. Two hours later there was progression of the numbness and a "feeling of choking to death." Shortly after this, examination revealed a sensory level at $\mathrm{T}-2$, paraplegia and severe weakness of the arm extensors, wrist extensors and flexors, and intrinsic hand muscles. Lumbar myelography demon-

- Dept. of Neurosurgery, University of Virginia Hospital, Charlottesville, Virginia, and Dept. of Anaesthesia, Faculty of Medicine, Memorial University of Newfoundland, St. John's, Newfoundland, Canada. 
strated a block to flow of contrast material at the L-2 level while cisternal myelography demonstrated a block at c-3. A two team cervical and lumbar laminectomy was then performed, revealing a large liquid epidural haematoma in which no clotted material was detected. This was presumed to have extended from $\mathrm{C}-3$ to $\mathrm{L}-2$, as communication between the laminectomy sites was established by injecting indigo carmine at the cervical laminectomy site and prompt recovery at the lumbar laminectomy site. Postoperatively the sensory level had dropped to the D-8-9 level bilaterally without any further change demonstrated on neurological examination. Tracheostomy became necessary because of severe respiratory insufficiency secondary to loss of function of accessory respiratory muscles. Myelography was repeated on the first postoperative day using the dye remaining in the subarachnoid space. This study showed no obstruction to flow present in the spinal canal. No further change in neurological status was noted.

A left above-knee amputation was required 14 days after the first operation because of progressive dry gangrene. The patient's condition gradually deteriorated with development of bilateral bronchopneumonia which was responsible for the patient's death on the 25 th hospital day.

\section{Previous Reports}

The earliest reported case of spontaneous epidural haematoma was made by Jackson in 1869.4 A 14-year-old girl developed progressive quadriparesis and eventually died in respiratory failure. The cause of the quadriparesis was found at necropsy to be a large cervical epidural haematoma. The diagnosis, incidentally, was made premortem by Sir William Jenner on the basis of the history and clinical findings. This entity has become well recognized, with over fifty cases reported in the literature to the present time.

The first case of epidural haematoma associated with anticoagulation was reported in $1952^{5}$ at which time Devanney and Osher at the Clinical Pathological Conference of the Cincinnati General Hospital discussed a case of a 62-year-old man receiving dicoumerol therapy for acute thrombophlebitis. The onset of low back pain was followed by radicular pain down the posterior aspect of both lower extremities. This was later accompanied by urinary retention, numbness, and weakness of the legs. Myelography revealed a block in the lumbar region. Surgery was delayed for seven days because of low prothrombin time. At the time of laminectomy, an epidural clot was found overlying the second to fifth lumbar segments. This was removed and there was eventually almost complete recovery.

The first case report of epidural haematoma associated with anticoagulation and continuous epidural catheterization was made in 1952 by Frumin and Schwartz ${ }^{1}$ describing a patient who received continuous lumbar epidural block for removal of a femoral embolus and treatment of postoperative arterial spasm. A haematoma formed in the epidural space during anticoagulation therapy. Prolonged loss of function of the lower extremities resulted from cord compression. Bromage ${ }^{2}$ described the second case, which was of a 72-year-old man who had his left femoral artery explored for presumed femoral embolus. The procedure was carried out under epidural block and an epidural catheter was inserted to ensure a 
continuous sympathetic block for several days. The patient was heparinized to prevent further clot formation. Thirty-six hours after the operation the patient died of a massive haemorrhage into the left leg. At postmortem examination, a large haematoma was present in the epidural space. Gingrich ${ }^{3}$ reported the case of a 72-year-old man with history of arteriosclerotic vascular disease and of having a Dacron prosthesis inserted at the aortoiliac bifurcation three years prior to admission. An epidural catheter was placed at the L-3-4 space to produce a chemical sympathectomy in an attempt to salvage as much of the leg as possible. Prior to amputation lidocaine was injected through the catheter. Sixteen hours after placement of the catheter anticoagulation therapy was started with the intravenous administration of heparin every four hours. At the forty-eighth hour the patient developed severe pain in the lumbar area, at which time the catheter was removed. During the next six hours he developed paraparesis and a sensory level at $\mathrm{T}-10$. He was taken to surgery where a haematoma of approximately $100 \mathrm{cc}$ was evacuated from the $\mathrm{T}-11-\mathrm{L}-4$ levels in the epidural space. During the next several months the patient recovered most of the sensory and motor deficit in the lower extremities. A left above-knee amputation was performed during this time. As an indication of the relative frequency of this complication, Lund ${ }^{6}$ in a survey of complications in 150,000 epidural blocks mentions "several reports" of epidural haematoma caused by continuous epidural catheters in patients who were receiving anticoagulation therapy.

\section{Pathological Anatomy}

Batson $^{\bar{T}}$ and Norgore ${ }^{8}$ showed that the posterior vertebral and epidural veins are of the primitive type having no valves and carrying blood at a pressure considerably lower than that in the venous system. Rupture of these veins leads to extravasation of blood into the loose network of epidural areolar and adipose tissue. The haematoma most commonly occupies a dorsal ${ }^{9,10}$ position in relation to the dural sac and usually extends over several vertebral segments. As in other space-occupying lesions, prompt removal is certainly indicated in order to relieve the neurological deficit.

\section{Discussion}

Winer et al. ${ }^{11}$, reviewed all cases of spontaneous epidural haematomata reported to that time and found that approximately one-fourth of the cases in the literature had coagulation defects, including those with blood dyscrasias. In his review of the literature on spontaneous epidural haematoma, Gold ${ }^{12}$ found that in onequarter of the reported cases coagulation defects had been present, mostly in elderly patients on long-term anticoagulation therapy. Spurning ${ }^{13}$ reviewed 43 cases of spinal epidural haematoma, eight of which were associated with anticoagulation therapy. In none of these reviews was an instance described of haematoma accompanying the use of epidural catheter and anticoagulation therapy.

Standard textbooks and monographs on the subject of epidural analgesia rather uniformly advise that an increased bleeding or clotting time secondary to the 
administration of anticoagulation is usually considered to be a contraindication of epidural analgesia, especially if the continuous technique is used. Considering the practicality of this, one must also weigh carefully the common use of epidural analgesia in many conditions where anticoagulation must be expected. The frequency of use of epidural analgesia in peripheral vascular surgery both for sympathectomy effect and operative procedures, where heparin administration has become an accepted mode of therapy, is well known. Its use in patients with known or unknown blood dyscrasias and in patients who are anticoagulated after myocardial infarction is also well recognized. Whether an increased bleeding or clotting time, no matter what the source, can be considered a contraindication to epidural anaesthesia will depend on the real incidence of epidural haematoma associated with this technique. If the real incidence of this complication were several times the reported incidence, a re-evaluation of this procedure in anticoagulated patients would most definitely be in order. If we can accept the incidence as reported, a policy of constant awareness of this complication would seem appropriate.

In an anticoagulated patient in whom an epidural catheter had been placed, detailed and frequent neurological examinations must be performed with this complication in mind. An informed and alert nursing staff must be aware of the signs and symptoms common to this clinical entity in its earliest stage. At the first complaint of severe back pain, paresthesia, lower extremity pain, numbness or weakness, the catheter must be removed and prompt neurosurgical evaluation must be done. Appropriate radiographic examination should then be performed if the question of epidural haematoma is raised and surgical intervention carried out if the diagnosis is established by this examination. Only by careful consideration of this complication at all times during the use of epidural analgesia in anticoagulated patients will this unfortunate complication be thwarted or recognized at a stage early enough to enable the surgeon to afford adequate decompression.

In reference to the surgical procedure in our case, a very small laminectomy was necessary, both in the cervical and lumbar areas, to evacuate the large liquid epidural haematoma. No solid clot was encountered which would probably have required a much more extensive procedure to afford adequate decompression.

\section{SUMMARY}

A case is presented of a 70-year-old man who developed progressive quadriplegia associated with epidural catheterization while on anticoagulation therapy. At operation a large liquid epidural haematoma was found extending from $\mathrm{C}-3$ to $\mathrm{L}-2$. Essentially no recovery of function was noted to time of death.

If epidural anaesthesia is to be used in anticoagulated patients, there must be recognition of the signs of onset of neurological complications so that surgical therapy is instituted immediately rather than after a delay of several hours.

\section{RÉSUMÉ}

On cite le cas d'un homme de 70 ans qui a présenté une quadriplégie progressive au cours d'un cathétérisme épidural alors que ce malade était soumis à un traite- 
ment anticoagulant. A l'opération on a trouvé un hématôme liquide considérable qui s'étendait de C-3 à L-2. On n'a noté aucun retour de fonction jusqu'à la mort du patient.

Si on doit utiliser l'anesthésie épidurale chez les malades soumis aux anticoagulants, il faut noter précocement les signes de complications neurologiques afin que le traitement chirurgical soit pratiqué immédiatement plutôt qu'après un délai de plusieurs heures.

\section{REFERENCES}

1. Frumin, M. J. \& Scrwartz, H. Continuous segmental peridural anaesthesia. Anaesthesiology. 13: 488 (1952).

2. Bromage, P. R. Spinal epidural analgesia. Baltimore: Williams and Wilkins (1954), pp. 101-102.

3. GiNGrich, T. F. Spinal epidural haematoma following continuous epidural anaesthesia. Anaesthesiology. 29: 162 (1968).

4. JaCkson, R. Case of spinal apoplexy. Lancet. 2: 5 (1869).

5. AqING, C. D. Neurological clinical pathological conference of the Cincinnati General Hospital, Diseases of the Nervous System. 13: 53 (1952).

6. Lund, P. C. Peridural anaesthesia. First ed. Springfield, Illinois: Charles C. Thomas (1966).

7. BAtson, O. V. The vertebral venous system as a mechanism for the spread of metastases. Amer. J. Roentgen. 48: 715 (1942).

8. Norgone, M. Clinical anatomy of vertebral veins, Surgery. 17:606 (1945).

9. Lougheed, W. M. \& Hoffman, H. J. Spontaneous extradural haematoma. Neurology. 10: $1059(1960)$.

10. LIN, T. H. Paraplegia caused by epidural hemorrhage of the spine. J. Int. Coll. Surg. 36: $742(1961)$.

11. Winer, B. M.; Horenstein, S.; \& Starr, A. M. Spinal epidural haematoma during anticoagulation therapy. Circulation. 19: 735 (1959).

12. Gold, M. E. Spontaneous spinal epidural haematoma. Radiology. 80: 823 (1963).

13. Spurninc, O. M. Spinal epidural haematoma-two cases. Arch. Intern. Med. 114: 103 (1964). 